\title{
Epistaxis: Its Prevalence in IDPs of North Waziristan Agency
}

\author{
Mohammad Iqbal ${ }^{1}$, Wasim Ahmed ${ }^{2, *}$ \\ ${ }^{1}$ Department of Otolaryngology, Head and Neck Surgery, Bannu Medical College, Bannu, KPK-Pakistan \\ ${ }^{2}$ Department of Biotechnology, Faculty of Biological Sciences, University of Science and Technology, Bannu, KPK-Pakistan
}

Email address:

waseem_bnu57@yahoo.com (W.Ahmed)

\section{To cite this article:}

Mohammad Iqbal, Wasim Ahmed. Epistaxis: Its Prevalence in IDPs of North Waziristan Agency. American Journal of Clinical and Experimental Medicine. Vol. 3, No. 5, 2015, pp. 233-236. doi: 10.11648/j.ajcem.20150305.16

\begin{abstract}
The objective of the aforesaid study was to assess the frequency of Epistaxis in IDPs who migrated from North Waziristan agency during military operation "Zarb-E-Azab". This study was conducted in ENT department, DHQ teaching hospital Bannu from august 2014 to May 2015. The inclusion criteria was acute epistaxis occurred due to any reason. The exclusion criteria were the natural blood stoppage. The data was collected based on age, gender, causes, type, treatment success rate and the actual need for hospitalization of victims. The results of this study showed that out of 121 patients during 10 months, $72 \%$ (87.12) were male patients while rest of the patients i.e. 28\% (33.88) were female. The prevalence of epistaxis in male was higher than the female patients. The average age group was 37 years. During the study, the most prevailing cause for the epistaxis was trauma $(50.20 \%)$ in youngsters which was followed by hypertension $(29.30 \%)$ in adults. Anterior epistaxis was around $58.20 \%$ while posterior one was $22.11 \% . \mathrm{AgNO}_{3}$ cautry $(56.45 \%)$ was the commonest treatment regimen followed by anterior nasal packing (32.78\%). We achieved a success rate of $30 \%$ for $\mathrm{AgNO}_{3}$ cautry and $47 \%$ for anterior nasal packing. In cases of acute/persistent epistaxis, where first-aid events have failed, and providing a bleeding vessel is visible, cautery with a silver nitrate tipped stick is the treatment of choice. However, where there is profuse bleeding from larger nasal arteries or veins, difficulty is often experienced in curtailing the blood flow, and commonly one must resort to electro cautry or tamponade by balloon or packing. Modifying the technique of silver nitrate cautry will result in universal success.
\end{abstract}

Keywords: Epistaxis, Electro Cautry, Silver Nitrate Cautry

\section{Introduction}

Epistaxis, or bleeding from the nose, is a communal grievance. It is rarely life threatening but may cause significant concern, especially among parents of small children.[1] Most nosebleeds are benign, self-limiting, and spontaneous, but some can be recurrent. Many uncommon causes are also noted.

Epistaxis can be divided into 2 categories, anterior bleeds and posterior bleeds, on the basis of the site where the bleeding originates. The true prevalence of epistaxis is not known, because most episodes are self-limited and thus are not reported. When medical attention is needed, it is usually because of either the recurrent or severe nature of the problem. Treatment depends on the clinical picture, the experience of the treating physician, and the availability of ancillary services.[2, 3, 4, 5] Bleeding typically occurs when the mucosa is eroded and vessels become exposed and subsequently break.

More than $90 \%$ of bleeds occur anteriorly and arise from Little's area, where the Kiesselbach plexus forms on the septum.[7, 8] The Kiesselbach plexus is where vessels from both the ICA (anterior and posterior ethmoid arteries) and the ECA (sphenopalatine and branches of the internal maxillary arteries) converge. These capillary or venous bleeds provide a constant ooze, rather than the profuse pumping of blood observed from an arterial origin. Anterior bleeding may also originate anterior to the inferior turbinate.

Posterior bleeds arise further back in the nasal cavity, are usually more profuse, and are often of arterial origin (eg, from branches of the sphenopalatine artery in the posterior nasal cavity or nasopharynx). A posterior source presents a greater risk of airway compromise, aspiration of blood, and greater difficulty controlling bleeding. 
Causes of epistaxis can be divided into local causes (eg, trauma, mucosal irritation, septal abnormality, inflammatory diseases, tumors), systemic causes (eg, blood dyscrasias, arteriosclerosis, hereditary hemorrhagic telangiectasia), and idiopathic causes. Local trauma is the most common cause, followed by facial trauma, foreign bodies, nasal or sinus infections, and prolonged inhalation of dry air. Children usually present with epistaxis due to local irritation or recent upper respiratory infection (URI).

In a retrospective cohort study of 2405 patients with epistaxis (3666 total episodes), Purkey et al used multivariate analysis to identify a series of risk factors for nosebleeds. The likelihood of epistaxis was found to increase in patients with allergic rhinitis, chronic sinusitis, hypertension, hematologic malignancy, coagulopathy, or, as mentioned, hereditary hemorrhagic telangiectasia. The investigators also found increased nosebleeds in association with older age and colder weather.[9]

\subsection{Trauma}

Self-induced trauma from repeated nasal picking can cause anterior septal mucosal ulceration and bleeding. This scenario is frequently observed in young children. Nasal foreign bodies that cause local trauma (eg, nasogastric and nasotracheal tubes) can be responsible for rare cases of epistaxis.

Acute facial and nasal trauma commonly leads to epistaxis. If the bleeding is from minor mucosal laceration, it is usually limited. However, extensive facial trauma can result in severe bleeding requiring nasal packing. In these patients, delayed epistaxis may signal the presence of a traumatic aneurysm.

Patients undergoing nasal surgery should be warned of the potential for epistaxis. As with nasal trauma, bleeding can range from minor (due to mucosal laceration) to severe (due to transection of a major vessel).

\subsection{Dry Weather}

Low humidity may lead to mucosal irritation. Epistaxis is more prevalent in dry climates and during cold weather due to the dehumidification of the nasal mucosa by home heating systems.

\subsection{Drugs}

Topical nasal drugs such as antihistamines and corticosteroids may cause mucosal irritation. Especially when applied directly to the nasal septum instead of the lateral walls, they may cause mild epistaxis. Medications such as nonsteroidal anti-inflammatory drugs (NSAIDs) are also frequently involved.

\subsection{Septal Abnormality}

Septal deviations (deviated nasal septum) and spurs may disrupt the normal nasal airflow, leading to dryness and epistaxis. The bleeding sites are usually located anterior to the spurs in most patients. The edges of septal perforations frequently harbor crusting and are common sources of epistaxis.

\subsection{Inflammation}

Bacterial, viral, and allergic rhinosinusitis causes mucosal inflammation and may lead to epistaxis. Bleeding in these cases is usually minor and frequently manifests as bloodstreaked nasal discharge.

Granulomatosis diseases such as sarcoidosis, Wegener granulomatosis, tuberculosis, syphilis, and rhinoscleroma often lead to crusting and friable mucosa and may be a cause of recurrent epistaxis. Young infants with gastroesophageal reflux into the nose may have epistaxis secondary to inflammation.

\subsection{Tumors}

Benign and malignant tumors can manifest as epistaxis. Affected patients may also present with signs and symptoms of nasal obstruction and rhinosinusitis, often unilateral.

Intranasal rhabdomyosarcoma, although rare, often begins in the nasal, orbital, or sinus area in children. Juvenile nasal angiofibroma in adolescent males may cause severe nasal bleeding as the initial symptom.

\subsection{Blood Dyscrasias}

Congenital coagulopathies should be suspected in individuals with a positive family history, easy bruising, or prolonged bleeding from minor trauma or surgery. Examples of congenital bleeding disorders include hemophilia and von Willebrand disease.[6]

Acquired coagulopathies can be primary (due to the diseases) or secondary (due to their treatments). Among the more common acquired coagulopathies are thrombocytopenia and liver disease with its consequential reduction in coagulation factors. Even in the absence of liver disease, alcoholism has also been associated with coagulopathy and epistaxis. Oral anticoagulants predispose to epistaxis.

\subsection{Vascular Abnormalities}

Arteriosclerotic vascular disease is considered a reason for the higher prevalence of epistaxis in elderly individuals. Hereditary hemorrhagic telangiectasia (HHT; is an autosomal dominant disease associated with recurrent bleeding from vascular anomalies. The condition can affect vessels ranging from capillaries to arteries, leading to the formation of telangiectasias and arteriovenous malformations. Pathologic examination of these lesions reveals a lack of elastic or muscular tissue in the vessel wall. As a result, bleeding can occur easily from minor trauma and tends not to stop spontaneously.

Various organ systems such as the respiratory, gastrointestinal, and genitourinary systems may be involved. The epistaxis in these individuals is variable in severity but is almost universally recurrent. Other vascular abnormalities that predispose to epistaxis include vascular neoplasms, aneurysms, and endometriosis. 


\subsection{Migraine}

Children with migraine headaches have a higher incidence of recurrent epistaxis than children without the disease.[10] The Kiesselbach plexus, which is part of the trigeminovascular system, has been implicated in the pathogenesis of migraine.[11]

\subsection{Hypertension}

The relationship between hypertension and epistaxis is often misunderstood. Patients with epistaxis commonly present with an elevated blood pressure. Epistaxis is more common in hypertensive patients, perhaps owing to vascular fragility from long-standing disease.

Hypertension, however, is rarely a direct cause of epistaxis More commonly, epistaxis and the associated anxiety cause an acute elevation of blood pressure. Therapy, therefore, should be focused on controlling hemorrhage and reducing anxiety as primary means of blood pressure reduction.

A study by Sarhan and Algamal, which included 40 patients with epistaxis and 40 controls, reported that the number of attacks of epistaxis was higher in patients with a history of hypertension, but the investigators were unable to determine whether a definite link existed between nosebleeds and high blood pressure. They did find, however, that control of epistaxis was more difficult in hypertensive patients; patients whose systolic blood pressure was higher at presentation tended to need management with packing, balloon devices, or cauterization.[12]

Excessive coughing causing nasal venous hypertension may be observed in pertussis or cystic fibrosis.

\subsection{Idiopathic Causes}

The cause of epistaxis is not always readily identifiable. Approximately $10 \%$ of patients with epistaxis have no identifiable causes even after a thorough evaluation.[13]

The current study was carried out in order to assess the frequency of the condition in IDPs of north Waziristan agency.

\section{Experimental}

The study was conceded in ENT department, DHQ teaching hospital district Bannu from august 2014 to May 2015. Inclusion criteria was acute epistaxis while exclusion criteria was natural blood stoppage. An otorynolarygological history and clinical examination was considered. Blood clot in the nasal area was lapped out. Drenched cotton plugs in $2 \%$ lidocaine and 1 ratio 1000 epinephrine were put into the nostrils for about 10 minutes in order to attain anesthesia along with vasoconstriction. Re-examination of the nose was done just after 10 minutes. Cross matching, $\mathrm{Hb}$ estimation was done by taking blood samples. Depending upon the severity and lethality of epistaxis, one or more of the following three conservative treatment modalities were used under local anesthesia; silver nitrate cautry (SNC), anterior nasal packing (ANP) or posterior nasal packing(PNP).
Initially, patients were treated with SNC, if the bleeding point was visible and unilateral. ANP was done with ribbon gauze impregnated with antibiotic ointment, in those patients where bleeding point was not visible and if the ooze was generalized or bleeding was profused. PNP was done with Folly's catheter, when the ANP did not work in controlling bleeding. Pack was left in for 24-48 hours. Spongeston (Gelfoam) was used in patients with bleeding diathesis, to avoid mucosal trauma associated with nasal packing.

Successful treatment was defined as, no recurrence of bleeding after SNC or removal of pack within one week. All those patients who re-bleeded after removal of pack, nasal packing was repeated and left for another 36 hours.

\section{Results \& Discussion}

Out of 121 patients of epistaxis during 10 months, $72 \%$ (87.12) were male patients while rest of the patients i.e. $28 \%$ (33.88) were female. The prevalence of epistaxis in male was higher than the female patients. The average age group was 37 years. During the study, the most prevailing cause for the epistaxis was trauma $(50.20 \%)$ in youngsters which was followed by hypertension $(29.30 \%)$ in adults. Anterior epistaxis was around $58.20 \%$ while posterior one was $22.11 \%$. $\mathrm{AgNO}_{3}$ cautry $(56.45 \%)$ was the commonest treatment regimen followed by anterior nasal packing $(32.78 \%)$. The results are shown in fig 1 . Similarly, different parameters are shown in table 1 including laterality, types and success rate of various treatment modalities.

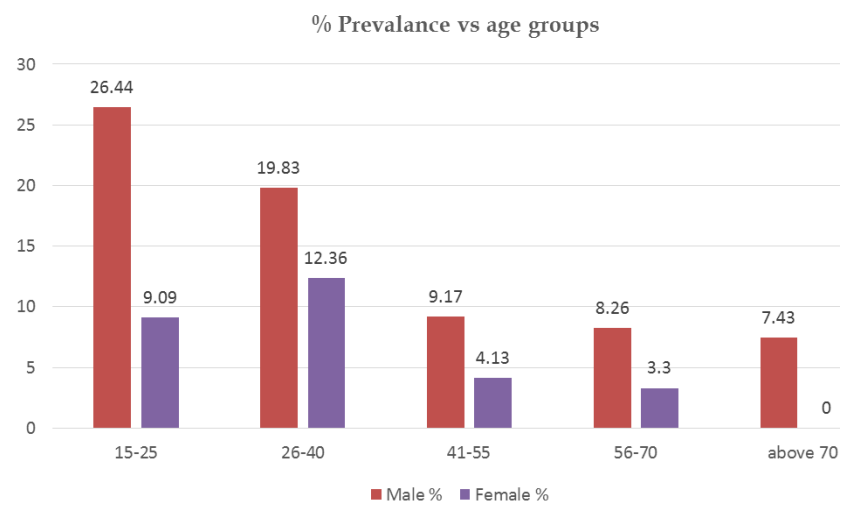

Fig. 1. \% prevalence of epistaxis in various age groups of male \& female.

Our results are according to the studies carried out previously $[14,15,16,17]$. The work of Awen et al showed all the victims belonging to pediatric group [18]. According to some studies, more than $75 \%$ of cases of nasal trauma present with epistaxis [19]. Trauma is involved in young age while epistaxis with hypertension was found in adult age in IDPs. Shaheen has reported that it is not the hypertension that causes epistaxis, rather it is the associated atherosclerosis that results in decreased vascular response to hemostasis and these patients tend to bleed heavily and longer [20]. Our study was limited to small no of patients but study with a larger population might help in understanding the various treatment regimens in management of epistaxis. 
Table 1. Different parameters of epistaxis in IDPs.

\begin{tabular}{lll}
\hline Characteristics & No of patients & \%age prevalence \\
\hline Anterior epistaxis & 79 & 65.28 \\
Posterior & 27 & 22.31 \\
Mixed & 15 & 12.39 \\
Total & 121 & \\
Unilateral & 91 & 75.20 \\
Bilateral & 30 & 24.79 \\
Total & 121 & \\
AgNO3 cautry & 76 & 62.80 \\
Anterior nasal packing & 25 & 20.66 \\
Posterior nasal packing & 14 & 11.57 \\
Spongeston packing & 6 & 4.95 \\
Total & 121 & \\
Success rate in: & & \\
AgNO3 cautery & $30 \%$ & \\
Anterior nasal packing & $47 \%$ & \\
Posterior nasal packing & $12 \%$ & \\
\hline
\end{tabular}

\section{References}

[1] Moreau S, De Rugy MG, Babin E, Courtheoux P, Valdazo A. Supraselective embolization in intractable epistaxis: review of 45 cases. Laryngoscope. 1998 Jun. 108(6):887-8.

[2] Abelson TI. Epistaxis. Schaefer SD. Rhinology and Sinus Disease 1st ed. New York: Mosby; 1998. 43-50.

[3] Douglas R, Wormald PJ. Update on epistaxis. Curr Opin Otolaryngol Head Neck Surg. 2007 Jun. 15(3):180-3.

[4] Emanuel JM. Epistaxis. Cummings CW. OtolaryngologyHead and Neck Surgery. 3rd ed. St. Louis: Mosby; 1998. 852865 .

[5] Pope LE, Hobbs CG. Epistaxis: an update on current management. Postgrad Med J. 2005 May. 81(955):309-14.

[6] Cummings CW. Epistaxis. Cummings. Otolaryngology: Head and Neck Surgery. 4th ed. Philadelphia, Pa: Elsevier, Mosby; 2005. Chap 40.

[7] Padgham N. Epistaxis: anatomical and clinical correlates. $J$ Laryngol Otol. 1990 Apr. 104(4):308-11.
[8] Guarisco JL, Graham HD 3rd. Epistaxis in children: causes, diagnosis, and treatment. Ear Nose Throat J. 1989 Jul. 68(7):522, 528-30, 532 passim.

[9] Purkey MR, Seeskin Z, Chandra R. Seasonal variation and predictors of epistaxis. Laryngoscope. 2014 Mar 15.

[10] Jarjour IT, Jarjour LK. Migraine and recurrent epistaxis in children. Pediatr Neurol. 2005 Aug. 33(2):94-7.

[11] Knight YE, Goadsby PJ. The periaqueductal grey matter modulates trigeminovascular input: a role in migraine? Neuroscience. 2001. 106(4):793-800.

[12] Sarhan NA, Algamal AM. Relationship between epistaxis and hypertension: a cause and effect or coincidence?. J Saudi Heart Assoc. 2015 Apr. 27 (2):79-84.

[13] Qureishi A, Burton MJ. Interventions for recurrent idiopathic epistaxis (nosebleeds) in children. Cochrane Database Syst Rev. 2012 Sep 12. 9:CD004461.

[14] Kucik CJ, Clenney T. Management of epistaxis summary for patients. Am Fam Physcian 2005;71:305-11.

[15] Pradhan. Management of refractory posterior epistaxis by sphenopalatine artery cauterization. Nepal J ENT Head Neck Surg 2010;1:3-5.

[16] Tevmoortash A, Sesterhenn A, Kress R, Sapundhiev N, Werner JA. Efficacy of ice packs in the management of epistaxis. Clin Otolaryngol Allied Sci 2003; 28:545-7.

[17] Hussain G, Iqbal M, Shah SA. Evaluation of aeitiology and efficacy of management protocol of epistaxis. J Ayub Med Coll Abbottabad 2006;18:62-5.

[18] Awan MS, Ali MM, Hussain I, Mian MY. Management of pediatrics epistaxis: a prospective study of 100 cases. Profess Med J 2001;8:226-65.

[19] Ali S, Mumtaz S, Saeed M. Epistaxis: aetiology and management. Ann King Edward Med Coll 2003;9:272-4.

[20] Shaheen OH. Epistaxis. In: Mackay IS, Bull TR, eds. Scott Brown's Otolaryngology. 5th edn London: Butterworths, 1987; 272-282. 